\title{
Type Ia supernovae as speed sensors at intermediate redshifts
}

\author{
Pengjie Zhang ${ }^{1,2}$ and Xuelei Chen $^{3}$ \\ ${ }^{1}$ Shanghai Astronomical Observatory, Chinese Academy of Science, 80 Nandan Road, Shanghai, China, 200030 \\ ${ }^{2}$ Joint Institute for Galaxy and Cosmology (JOINGC) of SHAO and USTC \\ ${ }^{3}$ National Astronomical Observatory, Chinese Academy of Science, Beijing, Chind
}

\begin{abstract}
Large scale peculiar velocity (LSPV) is a crucial probe of dark matter, dark energy and gravity at cosmological scales. However, its application is severely limited by measurement obstacles. We show that fluctuations in type Ia supernovae (SNe Ia) fluxes induced by LSPV offer a promising approach to measure LSPV at intermediate redshifts. In the 3D Fourier space, gravitational lensing, the dominant systematical error, is well suppressed, localized and can be further corrected effectively. Advance in SN observations can further significantly reduce shot noise induced by SN intrinsic fluctuations, which is the dominant statistical error. Robust mapping on the motion of the dark universe through SNe Ia is thus feasible to $z \sim 0.5$.
\end{abstract}

PACS numbers: 98.62.Py;98.80.-k;95.36.+x

\section{INTRODUCTION}

Matter distribution of the Universe is being revealed to great details by surveys on galaxies, gravitational lensing, the thermal Sunyaev Zel'dovich (SZ) effect, CMB, X-ray, etc. In contrast, measurements on large scale peculiar velocity (LSPV), or bulk flow, are still limited. Measurements which rely on distance indicators to subtract Hubble flow [1] are limited to local universe. Those based on anisotropic galaxy clustering in redshift space can be extended to cosmological distances [2]. However, this does rely on modeling of galaxy redshift distortion, whose accuracy still requires much improvement in this era of precision cosmology [3]. The kinetic SZ (KSZ) effect [4] of clusters is a promising probe [5], although systematic errors do exist [6]. Statistics of the diffuse KSZ background, such as the power spectrum [7] and cross correlations with other tracers of the large scale structure [8] can be measured robustly. However, this background measures the peculiar momentum instead and thus probes LSPV only indirectly.

LSPV, as a direct tracer of gravitational potential at cosmological scales, is powerful to probe dark matter, dark energy [9] and gravity [10]. In fact, it is indispensable to distinguish between some scenarios on the dark sectors [11]. We show that it is promising to measure LSPV robustly through type Ia supernovae (SNe Ia) and open a new window into the dark universe.

Peculiar velocity of a SN at position $\vec{x}$ shifts the apparent redshift to $1+z=(1+\bar{z})(1+\vec{v} \cdot \hat{x})$, where $\bar{z}$ is the real redshift. It also changes the luminosity distance to $d_{L}(z)=\bar{d}_{L}(\bar{z})(1+2 \vec{v} \cdot \hat{x})$. Fluctuations induced in its flux (with respect to the mean flux at $z$, instead of at $\bar{z}$ )

*Electronic address: pjzhang@shao.ac.cn,xuelei@cosmology.bao.ac.cn is then [12]

$$
\delta_{F}^{v}(\vec{x})=Q(z) \vec{v} \cdot \hat{x} ; Q(z)=-2\left(1-\frac{1+z}{\chi H}\right) .
$$

Here, $H$ is the Hubble constant at redshift $z$ and $\chi=$ $\int d z / H$ is the comoving distance. We have neglected the earth motion, whose effect is straightforward to take into account. The signal $\delta_{F}^{v} \sim v /(c z)$ is contaminated by $\delta_{F}^{L}$ induced by gravitational lensing ${ }^{1}$ and $\delta_{F}^{\text {random }}$ in-

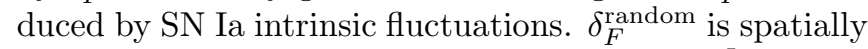
uncorrelated and straightforward to correct. $\delta_{F}^{L}$ induces systematical errors in velocity measurement, however, at local universe where $z<0.1$, contaminations of $\delta_{F}^{L}$ are negligible $\left(\left\langle\left(\delta_{F}^{v}\right)^{2}\right\rangle \gtrsim 10^{-4} \gg\left\langle\left(\delta_{F}^{L}\right)^{2}\right\rangle\right)$. These properties have enabled success in local LSPV measurements [14].

Gravitational lensing induced systematical error increases with redshift, while the signal decreases. So eventually the lensing induced systematical error will overwhelm the signal at intermediate redshifts. For example, at $z \sim 0.5, \delta_{F}^{v} \sim 0.1 \% \lesssim \delta_{F}^{L}$. If not corrected, this will prohibit the application of SNe Ia as cosmic speed censors. However, as the main result of this paper, we will show that, lensing induced systematical error has a intrinsically different pattern to that of $\delta_{F}^{v}$ and can be effectively corrected in the 3D Fourier space. On the other hand, shot noise induced by SNe Ia intrinsic fluctuations, which is the dominant statistical error, can be overcome by advance in observations. This approach does not rely on assumptions on SN Ia absolute luminosity, other than that its rms fluctuation is small, since it only explores information imprinted in flux fluctuations. Eventually,

\footnotetext{
${ }^{1}$ Dust extinction also causes fluctuations in SN fluxes. This kind of fluctuations has opposite sign to that of gravitational lensing and thus suppresses contaminations in velocity measurement. For cosmic gray dust, contaminations can be suppressed by 10$50 \%$ [13].
} 
high precision mapping of the motion of the dark universe to $z \sim 0.5$ will be realized through SNe Ia.

For a narrow redshift bin, $\delta_{F}^{v}$ and $\delta_{F}^{L}$ are virtually uncorrelated, due to the cancellation of positive $v$ and negative $v$ and the lensing weighting function. We then have $\left\langle\delta_{F}\left(\vec{x}_{1}\right) \delta_{F}\left(\vec{x}_{2}\right)\right\rangle=4 w_{\kappa}\left(\vec{x}_{1}, \vec{x}_{2}\right)+Q^{2}(z) \xi_{v}\left(\vec{x}_{1}, \vec{x}_{1}\right)$. Here, the direct observable is the total flux fluctuation (the sum of three)

$$
\delta_{F}=\delta_{F}^{v}+\delta_{F}^{L}+\delta_{F}^{\text {random }} .
$$

$w_{\kappa}\left(\vec{x}_{1}, \vec{x}_{2}\right) \equiv\left\langle\kappa\left(\vec{x}_{1}\right) \kappa\left(\vec{x}_{2}\right)\right\rangle$ and $\xi_{v}\left(\vec{x}_{1}\right) \equiv\left\langle\vec{v}\left(\vec{x}_{1}\right) \cdot \hat{x}_{1} \vec{v}\left(\vec{x}_{2}\right)\right.$. $\left.\hat{x}_{2}\right\rangle$ are the correlation functions of $\kappa$ and $\vec{v}(\vec{x}) \cdot \hat{x}$, respectively. $\kappa$ is the lensing convergence of a SN Ia at position $\vec{x}$ and $\delta_{F}^{L}=2 \kappa$ in the weak lensing regime. $w_{\kappa}\left(\vec{x}_{1}, \vec{x}_{2}\right)$ is anisotropic with respect to $\vec{x}_{1}-\vec{x}_{2}{ }^{2}$. It depends primarily on $\vec{\theta}_{12}$, the angular separation of two lines of sight (l.o.s.). The 2D Fourier transform over $\vec{\theta}_{12}$ (under the flat sky approximation) gives the usual 2D lensing power spectrum $C_{l}^{\kappa}\left(x_{1}, x_{2}\right)$. On the other hand, $w_{\kappa}\left(\vec{x}_{1}, \vec{x}_{2}\right)$ only weakly depends on $x_{1}-x_{2}$, the radial separation along the l.o.s.. Namely $w_{\kappa}$ lacks small scale power along the l.o.s. Correspondingly, the $3 \mathrm{D}$ power spectrum $P_{3 D}^{\kappa}(\vec{k})$, which is the 3D Fourier transform of $w_{\kappa}$, is highly anisotropic in the $3 \mathrm{D}$ wavevector $\vec{k}$ space. Its power should concentrate on a $2 \mathrm{D}$ plane of $\vec{k}$ perpendicular to the l.o.s., while its power along the l.o.s. can be significantly suppressed. On the other hand, $P_{3 D}^{v}(\vec{k})$, which is the $3 \mathrm{D}$ power spectrum of $\hat{v} \cdot \hat{x}$, has the opposite behavior. Its power concentrates along the l.o.s.. This brings hope to measure $P_{3 D}^{v}(\vec{k})$ along the l.o.s..

\section{THE 3D FLUX FLUCTUATION POWER SPECTRUM}

In the appendix, we derive the analytical expression of the 3D power spectra $P_{3 D}^{\kappa}(\vec{k})$ and $P_{3 D}^{v}(\vec{k})$. These results apply to survey volume in a narrow redshift range $[z-$ $\Delta z / 2, z+\Delta z / 2]$ and sufficiently small sky area $\theta_{\max } \times$ $\theta_{\max }$. We require $\Delta z \ll z$ and $\theta_{\max } / 2 \ll 90^{\circ}$ and choose the coordinate such that the center of the survey volume is $\vec{x}_{c}=\left(0,0, x_{c}\right)$. For the small sky coverage adopted, the l.o.s. is close to the direction to the survey center, which we denote with subscript $\|$. The directions perpendicular to the direction to the survey center are denoted with subscript $\perp$. Here we summarize the results.

\footnotetext{
2 During preparation of this work, A. Vallinotto et al. [15] and L. Hui et al. 16] published works on 3D lensing correlation function. Especially in [16] and a pioneer work [17] by T. Matsubara that [16] pointed out, the anisotropy in the correlation function $w_{\kappa}\left(\vec{x}_{1}, \vec{x}_{2}\right)$ is explicitly calculated. The result presented in this paper is performed in Fourier space, complementary to theirs and particularly suitable for LSPV reconstruction that this paper focuses on. Recently, Hui et al. [18] published their result in Fourier space, which is consistent with ours.
}

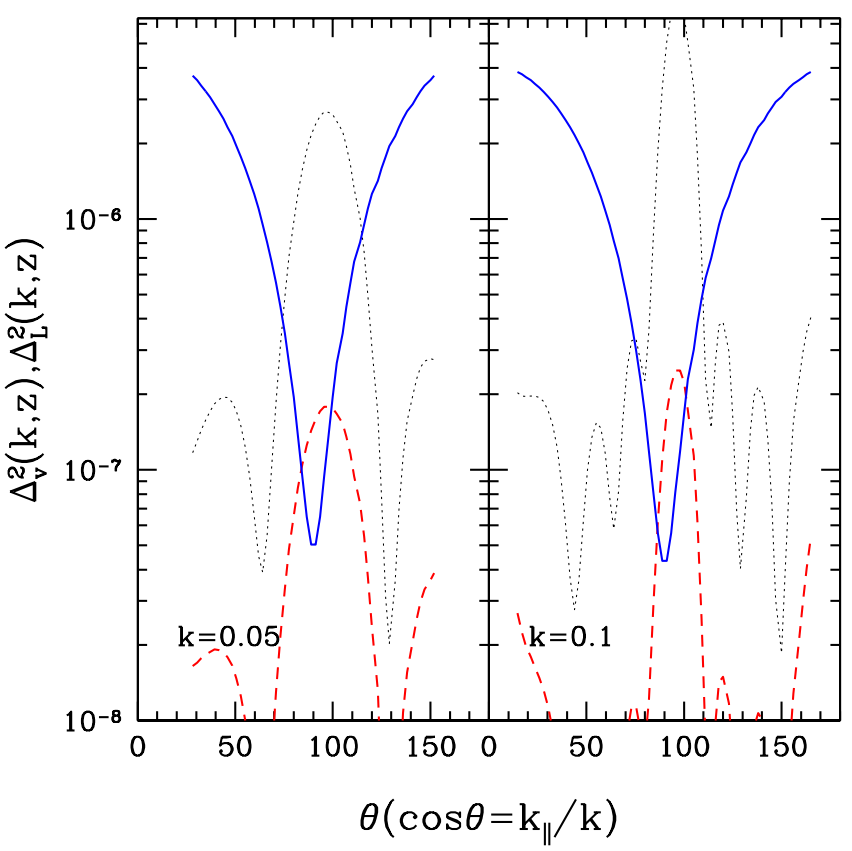

FIG. 1: The 3D power spectrum variance of SNe Ia flux fluctuations, which is the sum of $\Delta_{v}^{2}(\vec{k}) \equiv Q^{2}(z) k^{3} P_{3 D}^{v}(\vec{k}) /\left(2 \pi^{2}\right)$ (solid lines) and $\Delta_{L}^{2}(\vec{k}) \equiv 4 k^{3} P_{3 D}^{\kappa}(\vec{k}) /\left(2 \pi^{2}\right)$ (dot lines), at $z \in[0.45,0.55] . \quad k$ is in unit of $h / \mathrm{Mpc}$. Gravitational lensing lacks small scale power along the l.o.s., which causes the oscillating features. The same reason causes significant suppression in $\Delta_{L}^{2}(\vec{k})$ at $k_{\|} \gg 2 \pi / \Delta x$, where LSPV can be measured accurately in a model independent way. When $k_{\|} \rightarrow 0$, $\Delta_{L}^{2}(\vec{k})$ is barely suppressed and $\Delta_{v}^{2}(\vec{k})$ can not be measured for these modes. $\Delta_{L}^{2}$ begins to increase where $k_{\perp} \rightarrow 0$, caused by its dependence on $C_{l=k_{\perp} x_{c}}^{\kappa}$. Noticing that $\Delta_{L}^{2}(\vec{k})$ is slightly asymmetric with respect to $\theta \rightarrow \pi-\theta$. The ensemble average of $\Delta_{L}^{2}$ can be calculated from independent lensing surveys. After subtraction, gravitational lensing only induces statistical error (long dash lines), which is estimated combining all $\theta_{\max } \times \theta_{\max }$ patches in half sky.

The lensing convergence $\kappa$ is determined by the matter over-density $\delta$ projected along the l.o.s. through $\kappa(\vec{x})=$ $\int \delta\left(x_{L} \hat{x}\right) W\left(x_{L}, x\right) d x_{L}$. The lensing kernel $W\left(x_{L}, x\right)=$ $3 \Omega_{0} H_{0}^{2} x_{L}\left(1-x_{L} / x\right) / 2$ where the lens distance $x_{L}<x$ and zero otherwise. $w_{\kappa}\left(\vec{x}_{1}, \vec{x}_{2}\right), C_{l}^{\kappa}\left(x_{1}, x_{2}\right)$, the $3 \mathrm{D}$ matter power spectrum $P_{m}(k, z)$ and the $3 \mathrm{D}$ lensing power spectrum $P_{3 D}^{\kappa}(\vec{k})$ are related by the following equations,

$$
\begin{gathered}
w\left(\vec{x}_{1}, \vec{x}_{2}\right)=\int \frac{d^{2} l}{(2 \pi)^{2}} C_{l}^{\kappa}\left(x_{1}, x_{2}\right) e^{-i \vec{l} \cdot\left(\hat{x}_{1, \perp}-\hat{x}_{2, \perp}\right)}, \\
C_{l}^{\kappa}\left(x_{1}, x_{2}\right)=\int P_{m}\left(\frac{l}{x_{L}} ; z_{L}\right) \frac{W\left(x_{L}, x_{1}\right) W\left(x_{L}, x_{2}\right)}{x_{L}^{2}} d x_{L},
\end{gathered}
$$

and

$$
P_{3 D}^{\kappa}(\vec{k})=x_{c}^{2} \Delta x \bar{C}_{l=k_{\perp} x_{c}} \bar{I}(\vec{k})
$$


Here, $\Delta x$ is the distance interval between $z-\Delta z / 2$ and $z+\Delta z / 2 . \bar{C}_{l}$ is $C_{l}\left(x_{1}, x_{2}\right)$ averaged over the source distribution $x_{1,2} . \bar{I}(\vec{k})$ is an oscillating function of $k_{\|}$, induced by the Fourier transform along the direction of the survey center and reflecting the fact that $w\left(\vec{x}_{1}, \vec{x}_{2}\right)$ lacks small scale power along the l.o.s.. When $k_{\|} \gg k_{\perp} \theta_{\max }$ and $g \equiv k_{\|} \Delta x / 2 \gg 1, \bar{I} \simeq(\sin g / g)^{2} \ll 1$. Only when $k \rightarrow 0$, $\bar{I} \simeq 1$.

On the other hand, the 3D power spectrum of $\delta_{F}^{v}$ is

$$
P_{3 D}^{v}(\vec{k}, z) \simeq \frac{\beta^{2} H^{2} a^{2}}{c^{2}} \frac{P_{m}(k, z)}{k^{4}}\left(k_{\|}^{2}+k_{\perp}^{2} \frac{\left\langle\hat{x}_{\perp}^{2}\right\rangle}{2}\right) .
$$

Here $D$ is the linear density growth factor, $\beta \equiv$ $d \ln D / d \ln a$ and $P_{m}(k, z)$ is the matter density power spectrum at redshift $z$. The measured flux fluctuation power spectrum is the sum of two: $4 P_{3 D}^{\kappa}+Q^{2}(z) P_{3 D}^{v}$.

We show the two $3 \mathrm{D}$ power spectra at $z=0.5$ in figure 1. We only show those modes with $l=k_{\perp} x_{c} \geq 30$, where the flat sky approximation holds. The power of $P_{3 D}^{v}$ concentrates along the l.o.s., whereas that of $P_{3 D}^{\kappa}$ can be significantly suppressed. Clearly, for modes with sufficiently large $k_{\|}$, contribution from LSPV outweighs that from gravitational lensing by a large factor. The ensemble average of $P_{3 D}^{\kappa}$ can be predicted from $C_{l}^{\kappa}$, which will be measured to high precision by upcoming lensing surveys on the same sky. By subtracting it from the measured flux power spectrum, the systematic error $P_{3 D}^{\kappa}$ is converted into statistical error and one obtains an unbiased measure of $P_{3 D}^{v}$ (Fig. 11).

One still needs to overcome SNe Ia intrinsic fluctuations, which induce the dominant statistical error,

$$
\begin{aligned}
\frac{\Delta P_{3 D}^{v}}{P_{3 D}^{v}} & \simeq \quad \sqrt{\frac{(2 \pi)^{2}}{k^{2} \Delta k f_{\Omega} V}} \frac{\sigma_{\text {intr }}^{2}}{\bar{n}_{\mathrm{SN}}^{2} \Delta_{v}^{2} 2 \pi^{2} / k^{3}} \\
& =0.4\left(\frac{k}{0.05}\right)^{3 / 2} \frac{10^{-3}}{\bar{n}_{\mathrm{SN}}}\left(\frac{\sigma_{\text {intr }}}{0.1}\right)^{2} \frac{3 \times 10^{-6}}{\Delta_{v}^{2}}\left(\frac{10^{9}}{V} \frac{k}{\Delta k f_{\Omega}}\right)^{1 / 2} .
\end{aligned}
$$

Here, $f_{\Omega}$ is the fractional solid angle of $\mathbf{k}$ modes used for the analysis. $10 \% \mathrm{rms}$ flux fluctuation $\left(\sigma_{\mathrm{intr}}=0.1\right)$ corresponds to roughly $0.1 \mathrm{mag}$ rms dispersion in magnitude. Better calibration of SNe Ia and improvement over survey noise are likely able to reduce $\sigma_{\text {intr }}[19]$. A factor of 2 decrease in $\sigma_{\text {intr }}$ would relax the requirement of $\bar{n}_{\mathrm{SN}}$ by a factor of 4 or the sky coverage by a factor of 16 . Otherwise, successful measurement of LSPV requires (1) large sky coverage $f_{\text {sky }} \sim 1$ and (2) high SN number density $\bar{n}_{\mathrm{SN}} \sim 10^{-3}(h / \mathrm{Mpc})^{3}$. There are no fundamental obstacles to reach the above goals. The $\mathrm{SNe}$ Ia rate at $z=0.5$ is $\simeq 10^{-4}(h / \mathrm{Mpc})^{3} / \mathrm{yr}[20,21]$. So the survey must be performed over decades to reach $\bar{n}_{\mathrm{SN}} \sim 10^{-3}(h / \mathrm{Mpc})^{3}$. Drift scan using many telescopes of large field of view is able to detect all SNe Ia over a significant fraction of the sky. With advance in technology, such survey can be feasible and economical [22]. Follow up measurement on these $\sim 10^{6}$ spectroscopic redshifts is also feasible, since low resolution spectroscopy with $\sigma_{v} \sim 300 \mathrm{~km} / \mathrm{s}$ suffices. Even for these surveys, the LSPV measurement will be still limited to directions with sufficiently large $k_{\|}$, however, such measurements contain full information on the 3D power spectrum of $\vec{v}$ (not only $\vec{v} \cdot \hat{x}$ ), since it is isotropic in $\vec{k}$ space. Furthermore, since the power of the velocity power spectrum variance peaks at $k \sim 0.1 \mathrm{~h} / \mathrm{Mpc}$ for the standard cosmology, the bulk information of velocities can be measured.

Measuring LSPV through SNe Ia requires ambitious improvement over currently proposed SNe Ia surveys. It thus requires strong justification. Here we briefly address some impacts of such measurement on precision cosmology. For cosmology based on general relativity, dark matter and smooth dark energy, LSPV measurement helps to break parameter degeneracies. For example, the strength of weak gravitational lensing increases with dark matter density $\Omega_{m}$, because of more lens mass, and $\sigma_{8}$, because of stronger density fluctuations. Thus there exists a degeneracy between $\Omega_{m}$ and $\sigma_{8}$. This degeneracy can be broken by the lensing tomography, through which one can infer the evolution of the matter density field, which is more sensitive to $\Omega_{m}$ than to $\sigma_{8} \cdot{ }^{3}$ On the other hand, LSPV is very useful to break this degeneracy, since the strength of LSPV is very sensitive to evolution in the matter density field. A simple justification is as follows. In the linear regime, from the continuity equation $\dot{\delta}+\nabla \cdot \vec{v}=0$, we have $v(\vec{k}, z) \propto f \delta(\vec{k}, z)$ in the Fourier space, where $f \simeq \Omega_{m}(z)^{0.56}$. With the measurement of $\delta(\vec{k}, z)$ (or the power spectrum $P_{m}(k)$ ) from weak lensing and LSPV measurement, one can directly infer $f(z)$ and thus the value of $\Omega_{m}$ to break the $\Omega_{m}-\sigma_{8}$ degeneracy in weak lensing cosmology. LSPV information is also highly complementary to CMB. The strength of CMB is sensitive to the shape and amplitude of the initial fluctuations. LSPV measurement directly provides such kind of information and can thus significantly improve the CMB cosmological constraints. For other applications, refer to [9].

If our universe is indeed fully described by general relativity (GR), dark matter and smooth dark energy, cosmological probes other than LSPV can already put stringent constraints on dark matter density, dark energy density and the dark energy equation of state [24]. In this case, LSPV measurement does not provide fundamentally new information, due to the fixed (and known) relations between fluctuations in the metric, density and LSPV in such cosmology. However, the dark universe can be more complicated. If we are open to the possibilities that dark energy may be clustered, with non-negligible anisotropic stree, and gravity at cosmological scales may deviate from GR, LSPV containes independent and cru-

\footnotetext{
${ }^{3}$ In the linear regime, the matter density evolution does not depend on $\sigma_{8}$. But in the nonlinear regime, it does.
} 
cial information to that in flucutations of metric and density field. LSPV measurement can thus provide valuable information, such as the nature of gravity at cosmological scales [10]. Indeed, to distinguish between some dark energy models from some modified gravity models, it is indispensable to have LSPV measurements, as shown in [11.

There are several ways of measuring LSPV. LSPV measurement through SNe Ia is complementary to other methods. Among them, a promising one is the kinetic Sunyaev Zel'dovich (KSZ) effect of galaxy clusters. Ongoing and planned surveys such as the south pole telescope $(\mathrm{SPT})^{4}$ plus the dark energy surveys (DES) ${ }^{5}$ are able to measure the LSPV power spectrum to $10 \%$ statistical accuracy at large scales [,,$\underline{6}$ ]. Future all sky surveys can further reduce the statistical error by a factor of 3. However, what the cluster KSZ effect directly measures is the total KSZ flux $\propto M_{g} v$, instead of $v$. Here $M_{g}$ is the total gas mass. Some complexities, such as feedback, cooling, point source contamination and nonisothermal/non-spherical gas distribution, could bias the estimation on $M_{g}$ [6] and thus bias the LSPV measurement through the cluster KSZ effect. On the other hand, LSPV measurement through SNe Ia is free of such uncertainties, althrough it may suffer some other systematics. For example, if the intrinsic SNe Ia fluctuations are somehow correlated with the large scale structure (although quite unlikely), LSPV measurements will be biased by such correlation. Nonetheless, SNe Ia provide an independent, likely clean and potentially powerful method to measure LSPV. For all these reasons, surveys capable of measuring LSPV through SNe Ia would be very profitable scientifically.

\section{DISCUSSIONS}

Throughout the paper we adopt the flat sky approximation, which breaks where $l=k_{\perp} x_{c} \rightarrow 0\left(k_{\perp} \rightarrow 0\right)$. However, we will show in a companion paper that, the feature that the power of $\delta_{F}^{L}$ is significantly suppressed along the l.o.s. survives beyond the flat sky approximation and so as the proposed LSPV measurement method.

The same technique can be applied to fluctuations in the fundamental plane relation of galaxies, for which peculiar velocity also contributes. Dispersion in the fundamental plane is larger than that of in SNe Ia. However, higher galaxy number density may be reached in shorter observation period and the overall shot noise can be reduced.

\footnotetext{
${ }^{4}$ http://pole.uchicago.edu/

${ }^{5}$ http://www.darkenergysurvey.org/
}

As the summary, this paper shows in a proof of concept study that contaminations induced by gravitational lensing in SN peculiar velocity measurements can be efficiently and unbiasedly corrected in 3D Fourier space. Combining with advance in SNe Ia surveys, it is feasible to measure the large scale peculiar velocity robustly and map the motion of the dark universe.

We thank Neal Dalal, Yipeng Jing and Zheng Zheng for useful discussions. This work is supported by the one-hundred-talents program of CAS, the NSFC grant 10533010, 10533030, the NSFC Distinguished Young Scholar grant No. 10525314, the CAS grant KJCX3SYW-N2 and the 973 program grant No. 2007CB815401.

\section{APPENDIX}

We choose the coordinate such that the center of the survey volume is at $\left(0,0, x_{c}\right)$. The $3 \mathrm{D}$ vector pointing to an object is $\vec{x} \equiv x \hat{x} \equiv\left(x \hat{x}_{\perp}, x_{\|}\right)$. We adopt the flat sky approximation. Under this approximation, the solid angle element $d \Omega_{\hat{x}} \simeq d^{2} \hat{x}_{\perp}$ and the volume element $d V \equiv$ $x^{2} d x d \Omega_{\hat{x}} \simeq x^{2} d x d \hat{x}_{\perp}$. Also, $x_{\|} \simeq x$, accurate to better than $2 \%$ accuracy, for $20^{\circ} \times 20^{\circ}$ sky.

The 3D lensing power spectrum $P_{3 D}^{\kappa}(\vec{k})$ is defined as

$$
P_{3 D}^{\kappa}(\vec{k}) \equiv \int w_{\kappa}\left(\vec{x}_{1}, \vec{x}_{2}\right) \exp \left(i \vec{k} \cdot\left[\vec{x}_{1}-\vec{x}_{2}\right]\right) \frac{d V_{1} d V_{2}}{V} .
$$

Plug the $w_{\kappa}-C_{l}^{\kappa}$ relation (Eq. 3) into Eq. 8, integrate over $d^{2} \hat{x}_{1 \perp}$ and then $d^{2} l$, we have

$P_{3 D}^{\kappa}(\vec{k})=\int C_{l=k_{\perp} x_{1}}\left(x_{1}, x_{2}\right) e^{i\left(x_{1}-x_{2}\right)\left(k_{\|}+\vec{k}_{\perp} \cdot \hat{x}_{2, \perp}\right)} \frac{x_{1}^{2} d x_{1} d V_{2}}{V}$

Plug into the Limber integral for $C_{l}^{\kappa}$ (Eq. 4), integrate over $d x_{i=1,2}$ and then integrate over $d \hat{x}_{2 \perp}$, we have

$$
\begin{aligned}
P_{3 D}^{\kappa}(\vec{k}) & \simeq x_{c}^{2} \Delta x \int P_{m}\left(\frac{k_{\perp} x_{c}}{x_{L}}, z_{L}\right) \frac{d x_{L}}{x_{L}^{2}} \bar{W}^{2}\left(x_{L}\right) I\left(\vec{k}, x_{L}\right) \\
& \equiv x_{c}^{2} \Delta x \bar{C}_{l=k_{\perp} x_{c}} \bar{I}(\vec{k}) .
\end{aligned}
$$

Here we have replaced $P_{m}\left(\frac{k_{\lrcorner} x_{1}}{x_{L}}\right)$ with $P_{m}\left(\frac{k_{\lrcorner} x_{c}}{x_{L}}\right)$, since $P_{m}$ varies slowly with $k$ and $x_{1} \simeq x_{c}$ for narrow redshift bins. $\bar{W}\left(x_{L}\right) \equiv \int W x^{2} d x / \int x^{2} d x$ is the usual weighted lensing kernel. The suppression factor

$$
I\left(\vec{k}, x_{L}\right) \equiv \bar{W}^{-2}\left(x_{L}\right)\left\langle\left|\frac{\int_{x_{l}}^{x_{u}} W\left(x_{L}, x\right) \exp [i b x] x^{2} d x}{\int_{x_{l}}^{x_{u}} x^{2} d x}\right|^{2}\right\rangle .
$$

Here, $b \equiv k_{\|}+\vec{k}_{\perp} \cdot \hat{x}_{\perp}$ and $\langle\cdots\rangle$ is averaged over the solid angle $d^{2} \hat{x}_{\perp}$. The term $b x$ in the exponential is $\vec{k} \cdot \vec{x}$ under the flat sky approximation. This implies that, an orthogonal Fourier mode is along the actual l.o.s. (instead of along the direction to the survey center). The other orthogonal Fourier modes are $\vec{k}_{\perp}$, which is the Fourier 
transform of angular direction. $\bar{I}(\vec{k})$ is $I\left(\vec{k}, x_{L}\right)$ weighted through $x_{L} \leq x_{u}$ and its exact definition is given by the last relation in Eq. 9. $x_{l}$ and $x_{u}$ are the distance at $\bar{z}-\Delta z / 2$ and $\bar{z}+\Delta z / 2$, respectively.

When $x_{L} \lesssim x_{l}, I\left(\vec{k}, x_{L}\right)$ varies slowly with respect to $x_{L}$, because $W\left(x_{L}, x\right)$ in the bracket varies slowly with respect to $x \in\left[x_{l}, x_{u}\right]$ and cancels the one in $\bar{W}$. When $x_{L} \rightarrow x_{u}, I$ increases rapidly and approaches 1 . Since most lensing contributions come from $x_{L}<x_{l}$ and the lensing weighting $\left(W^{2} I\right)$ peaks at $x_{L} \simeq x_{u} / 2$, a convenient approximation is that $\bar{I}(\vec{k}) \simeq I\left(\vec{k}, x_{L}=x_{u} / 2\right)$. Numerical evaluation of Eq. 9 shows that this approximation degrades for smaller $k$, however, even for $\vec{k}=(0,0,0.05) h / \mathrm{Mpc}$, it still works to $10 \%$ accuracy. We will adopt this approximation, for its simplicity and clear physical meaning.

At linear scale, peculiar velocities are irrotational. The mass conservation equation $\dot{\delta}+\nabla \cdot \vec{v} / a=0$ then tells that $\vec{v}(\vec{k})=i \delta(\vec{k}) \vec{k} / k^{2}$. The $3 \mathrm{D}$ (l.o.s.) velocity power spectrum is

$$
\begin{aligned}
P_{3 D}^{v}\left(\vec{k}^{\prime}\right) \equiv & \int\left\langle\vec{v}\left(\vec{x}_{1}\right) \cdot \hat{x}_{1} \vec{v}\left(\vec{x}_{2}\right) \cdot \hat{x}_{2}\right\rangle e^{-i \vec{k}^{\prime} \cdot\left(\vec{x}_{1}-\vec{x}_{2}\right)} \frac{d V_{1} d V_{2}}{V} \\
\simeq & \int \frac{d^{3} k}{(2 \pi)^{3}} \frac{P_{m}(k, z)}{k^{4}} e^{i\left(\vec{k}-\vec{k}^{\prime}\right)\left(\vec{x}_{1}-\vec{x}_{2}\right)} \frac{d V_{1} d V_{2}}{V} \\
& \left.\left(k_{\|}^{2}+\left(k_{\perp} \cdot \hat{x}_{1, \perp}\right)\left(k_{\perp} \cdot \hat{x}_{2, \perp}\right)+2 k_{\|} k_{\perp} \cdot \hat{x}_{\perp}\right)\right) \\
= & \frac{P_{m}(k, z)}{k^{4}} k_{\|}^{2}+\int \frac{d^{3} k}{(2 \pi)^{3}} \frac{P_{m}(k)}{k^{4}} \frac{d V_{1} d V_{2}}{V} \\
& \left(\left(k_{\perp} \cdot \hat{x}_{\perp}\right)^{2}-\left(k_{\perp} \cdot \frac{\Delta \hat{x}_{\perp}}{2}\right)^{2}\right) e^{i\left(\vec{k}-\vec{k}^{\prime}\right)\left(\vec{x}_{1}-\vec{x}_{2}\right)}
\end{aligned}
$$

Here, $\hat{x}_{\perp} \equiv\left(\hat{x}_{1 \perp}+\hat{x}_{2 \perp}\right) / 2, \Delta \hat{x}_{\perp} \equiv \hat{x}_{1 \perp}-\hat{x}_{2 \perp}$ and $\vec{x}_{1, \perp}-$ $\vec{x}_{2, \perp} \simeq x_{c} \Delta \hat{x}_{\perp}$. Finally we have

$$
\begin{aligned}
P_{3 D}^{v}\left(\vec{k}^{\prime}\right) & =\frac{\beta^{2} H^{2} a^{2}}{c^{2}} \frac{P_{m}(k, z)}{k^{4}} k_{\|}^{2}+\frac{\beta^{2} H^{2} a^{2}}{c^{2}} \\
& \times\left(\frac{P_{m}(k, z) k_{\perp}^{2}\left\langle\hat{x}_{\perp}^{2}\right\rangle}{2 k^{4}}+\left[\frac{P_{m}(k, z) k_{i} k_{j}}{k^{4} 4 x_{c}^{2}}\right]_{, i j}\right) .
\end{aligned}
$$

Nonlinearity at small scales could modify the above result. For example, shell crossing generates vorticity (rotational velocity component), whose impact on $P_{3 D}^{v}(\vec{k})$ could be non-negligible where $\vec{k}_{\perp} \neq 0$. However, such possible modifications do not invalidate the proposed LSPV measurement approach. Since the main purpose of this paper is to demonstrate the feasibility of measuring LSPV through SNe Ia, instead of developing an advanced LSPV model, Eq. 10] suffices. Furthermore, due to the extra factor $\left(k x_{c}\right)^{-2}$, the last term in Eq. 10 is much smaller than the second term for relevant $k$ and will be neglected elsewhere in this paper.
[1] e.g., M. J. Hudson et al., MNRAS 352, 61 (2004); D. Sarkar et al., MNRAS 375, 691 (2007)

[2] M. Tegmark, A. Hamilton and Y. Xu, MNRAS 335, 887 (2002); M. Tegmark et al. Astrophys.J. 606 (2004) 702

[3] R. Scoccimarro PRD 70, 083007 (2004)

[4] R. Sunyaev and Y. B. Zel'dovich, MNRAS 190, 413 (1980).

[5] G. Holder, ApJ 602, 18 (2001); F. Atrio-Barandela, A. Kashlinsky, and J. P. Mucket,ApJL 601, L111 (2004); P.J. Zhang, H. Feldman, R. Juszkiewicz \& A. Stebbins, 2008, MNRAS, in press.

[6] N. Aghanim, S. Hansen, and G. Lagache, A\&A 439, 901A (2005); A. Diaferio, et al., MNRAS 356, 1477 (2005).

[7] E. T. Vishniac, ApJ 322, 597 (1987); W. Hu, ApJ 529, 12 (2000); C-P. Ma and J. N. Fry, PRL 88, 211301 (2002); P. J. Zhang, U. L. Pen and B. Wang, MNRAS 347, 1224 (2004)

[8] O. Doré, J. F. Hennawi, and D. N. Spergel. ApJ 606, 46 (2004)

[9] e.g. H. A. Feldman et al., ApJL 596, L131 (2003a); P. Fosalba and O. Doré, arxiv:astro-ph/0701782; C. Gordon, K. Land, and A. Slosar, arxiv:0705.1718; Bhattacharya \& Kosowsky 2006 ApJL, 659, L83. astro-ph/0612555

[10] e.g. P.J. Zhang, M. Liguori, R. Bean, and S. Dodelson, arxiv:0704.1932; Eric V. Linder. arXiv:0709.1113

[11] Bhuvnesh Jain, Pengjie Zhang. arXiv:0709.2375

[12] Hui, L. and P. B. Greene, PRD 73, 123526 (2006).

[13] P.J. Zhang, and P.S. Corasaniti. ApJ 657, 71 (2007).

[14] e.g. C. Bonvin, R. Durrer, \& M. Kunz, PRL 96, 191302 (2006); T. Haugboelle et al., arxiv:astroph/0612137; R. Watkins, and H. A. Feldman, arxiv:astroph/0702751; L. F. Wang, arxiv:0705.0368.

[15] A. Vallinotto, S. Dodelson, C. Schimd, and J-P. Uzan, arxiv:astro-ph/0702606.

[16] L. Hui, E. Gaztanaga, and M. LoVerde, arxiv:0706.1071.

[17] T. Matsubara, ApJL 537, L77 (2000).

[18] Lam Hui, Enrique Gaztanaga, Marilena LoVerde. 2007, arXiv:0710.4191

[19] X.F. Wang, L.F. Wang, X. Zhou, Y.Q. Lou, Z.W. Li. Astrophys.J. 620 (2005) L87 astro-ph/0501565.

[20] Dovi Poznanski et al. arXiv:0707.0393

[21] N. Kuznetsova et al. arXiv:0710.3120 The SNe Ia rate in this paper is in unit of per year per $\left(\mathrm{Mpc} / h_{70}\right)^{3}$. With $H=70 \mathrm{~km} / \mathrm{s} / \mathrm{Mpc}$, we need to multiply by $\left(h_{70} / h\right)^{-3}$ to get the SNe Ia rate in unit of per year per $(\mathrm{Mpc} / h)^{3}$. The result at $z=0.5$ is consistent with [20].

[22] We only briefly mention one design which may fulfill the survey requirement. ALPACA [23], a proposed 8 meter rotating liquid mercury mirror telescope for $\mathrm{SNe}$ Ia search, has $\sim 10 \mathrm{deg}^{2}$ field of view. $\sim 100$ of such telescopes can be constructed with reasonably low cost and detect all SNe Ia over the whole sky.

[23] P.S. Corasaniti, M. LoVerde, A. Crotts, C. Blake. Mon.Not.Roy.Astron.Soc. 369 (2006) 798. astro-ph/0511632; http://www.astro.ubc.ca/LMT/alpaca http://www.itp.ac.cn/Download/spac2005/ArlinCrotts.ppt 
[24] For a comprehensive review, refer to the Report of the Dark Energy Task Force (Andreas Albrecht, et al. arXiv:astro-ph/0609591) 